\title{
Plasma neurofilament light chain concentration is increased and correlates with the severity of neuropathy in hereditary transthyretin amyloidosis
}

Kapoor $\mathrm{M}^{1}$, Foiani $\mathrm{M}^{2,3}$, Heslegrave $\mathrm{A}^{2,3}$, Zetterberg $\mathrm{H}^{3,4,5}$, Lunn $\mathrm{MP}^{1}$, Malaspina $\mathrm{A}^{6}$, Gillmore $\mathrm{J}^{7}$, Rossor $\mathrm{AM}^{1^{*}}$, Reilly $\mathrm{MM}^{1^{*}}$

1. MRC Centre for Neuromuscular Diseases, Department of Neuromuscular Diseases, UCL Queen Square Institute of Neurology, London, United Kingdom.

2. Department of Neurodegenerative Disease, University College London Queen Square Institute of Neurology, London, United Kingdom.

3. UK Dementia Research Institute at University College London, London, United Kingdom.

4. Clinical Neurochemistry Laboratory, Sahlgrenska University Hospital, Mölndal, Sweden

5. Department of Psychiatry and Neurochemistry, Institute of Neuroscience and Physiology, Sahlgrenska Academy at University of Gothenburg, Mölndal, Sweden.

6. Trauma and Neuroscience Centre, Blizard Institute, Barts and the London School of Medicine and Dentistry, Queen Mary University of London, London, UK

7. National Amyloidosis Centre, University College London, Royal Free Campus, London, UK

Running headline: Plasma neurofilament light chain in ATTR

*equal contribution

Word Count: 1186

Tables: 1

Figures: 3

Corresponding author:

Alexander M Rossor. Department of Neuromuscular Diseases, University College London Queen Square Institute of Neurology, London, United Kingdom. A.rossor@ucl.ac.uk 


\begin{abstract}
Hereditary transthyretin amyloidosis (ATTRm) causes a disabling peripheral neuropathy as part of a multi-system disorder. The recent development of highly effective gene silencing therapies has highlighted the need for effective biomarkers of disease activity to guide the decision of when to start and stop treatment. In this study we measured plasma neurofilament light chain (pNfL) concentration in 73 patients with ATTR and found that pNfL was significantly raised in ATTRm patients with peripheral neuropathy compared to healthy controls. Furthermore, pNFL correlated with disease severity as defined by established clinical outcome measures in 26 patients for whom this information was available. These findings suggest a potential role of $\mathrm{pNfL}$ in monitoring clinical progression in ATTRm patients.
\end{abstract}

\title{
Keywords:
}

Neurofilament light chain

Hereditary transthyretin amyloidosis

Biomarkers

Neuropathy impairment Score

Peripheral neuropathy 


\section{Introduction}

Hereditary transthyretin amyloidosis is an aggressive, multisystem disorder due to mutations in the TTR gene that promote the deposition of TTR amyloid with a propensity for the heart and peripheral and autonomic nervous systems ${ }^{1}$. More than $90 \%$ of TTR is synthesised in the liver. As TTR is a relatively redundant protein, therapeutic strategies have focused on supressing total TTR production from the liver in an effort to reduce the rate of amyloid deposition and disease progression. In 2018, two genetic therapies (an antisense oligonucleotide and a RNAi) both achieved their primary outcome measures in a trial of ATTRm peripheral neuropathy and have been approved by the FDA and $\mathrm{EMA}^{2,3}$. As ATTRm has a wide range of age of onset with some mutations not being fully penetrant. One of the most pressing issues in the current management of ATTRm is both to diagnose ATTRm as early as possible and to define the optimum time to start gene silencing therapy. Clinical examination and outcome measures, such as the neuropathy impairment score (NIS), will detect the development of a significant large fibre neuropathy but unfortunately, by the time the neuropathy is clinically evident, the disease often progresses rapidly and irreversible disability is acquired ${ }^{4}$.

There is therefore a need for a biomarker that can detect the onset of peripheral neuropathy before the emergence of significant clinical disability. Neurofilaments are the major cytoskeletal proteins of neurons and comprise a light, medium and heavy chain. Following axonal damage, neurofilaments are released into the circulation and can be detected in the plasma using highly sensitive analytical methods such as the SIMOA platform ${ }^{5}$. We have previously shown that pNFL is raised in patients with Charcot-Marie-Tooth (CMT) disease and that it correlates with disease severity ${ }^{6}$. In this study we sought to determine whether pNFL was also raised in patients with ATTRm neuropathy compared to controls and if it correlates with disease severity. 


\section{Subjects and Methods}

Blood samples were collected from neurologically symptomatic and asymptomatic ATTRm patients attending the UK National Amyloid Centre and the National Hospital for Neurology and Neurosurgery. Control samples were collected from spouses or friends of patients as part of a separate study. The severity of the neuropathy was recorded using the Rasch modified CMT examination and the neuropathy impairment scores within four months of plasma collection $^{7,8,9}$. This study was approved by the NHNN Research Ethics Committee (REC)/ Central London (09/H0716/61), the East London and the City REC (09/H0703/27) and Royal Free Hospital and University College Medical School REC (06/Q0501/42). Written informed consent was obtained from all participants in the study. Genetic testing was performed according to standard protocols (details are available upon request).

\section{Laboratory Markers}

Blood was collected into EDTA-containing tubes, centrifuged at $20^{\circ} \mathrm{C}$ at $3,500 \mathrm{rpm}$ for 10 minutes and stored within 1 hour at $-80^{\circ} \mathrm{C}$ until analysis. Plasma NfL concentration was determined using an in-house Simoa NfL assay, as previously described ${ }^{10}$.

\section{Statistical Analysis}

Plasma NfL concentration and age was compared using a 2-sided Mann-Whitney U test and correlations were determined using Pearson's and Spearman's correlation co-efficient, at a significance level of 5\%. Multiple linear regression was performed to model the relationship between pNfL (dependent variable), age, NIS and CMTES-R score (independent variables). Statistical analysis was performed using SPSS version 24 (IBM; Armonk, NY) and GraphPad Prism 8.0.1 (GraphPad Inc., La Jolla, CA). 


\section{Results}

A total of 73 patients with pathogenic TTR mutations (V30M n=49, T60A n=10, S77Y n=22) and 16 healthy controls were enrolled in to the study. There was a significant difference in the mean ages $(T T R=56$ years, controls $=67$ years; $p=0.04)$ of the 2 groups $($ Table 1$)$. Five patients were taking diflunisal for a median time of 127 days (range 60-2379 days), 1 patient was on tafamidis. 26 patients had disease severity measured (MRC sum score, NIS and/or CMTES-R) within 4 months of blood collection (Table 1).

Plasma NfL concentration was significantly higher in the entire group of patients with ATTRm, and in the subgroup of ATTRm patients with a neuropathy compared to healthy controls $(\mathrm{p}<0.001$; Figure 1). An ANCOVA was run to determine whether $\mathrm{pNfL}$ differed between healthy controls and those ATTRm patients with no clinical neurological findings ( six participants, NIS=0, MRC=70, CMTES-R=0) after controlling for age. After adjustment for age, there was no statistically significant difference in $\mathrm{pNfL}, \mathrm{F}(1,23)=0.70, \mathrm{p}=0.41$, partial $\eta 2=.03$

Multiple regression analysis to predict pNfL concentration from age, NIS, and CMTES-R found that disease severity measured by NIS was a significant predictor $(\beta=1.167, \mathrm{p}=$ 0.006,), whereas age and CMTES-R were not (age: $\beta=0.04, p=0.94$; CMTES-R: $\beta=2.767$, $\mathrm{p}=0.057$, .). A Spearman's rank-order correlation was run to assess the relationship between NIS and pNfL, and CMTES-R and pNfL There was a statistically significant, positive correlation between NIS and pNfL (rs $(30)=0.65, \mathrm{p}<0.0001$, Figure $2 \mathrm{a}$ ) and CMTES-R and pNfL (rs $(35)=0.69, \mathrm{p}<0.0001$, Figure $2 \mathrm{~b})$ ). Correlation analysis between NIS and

CMTES-R showed a tight interaction between these 2 scores $(r=0.89, p=<0.0001$, Figure $3)$. 


\section{Discussion}

In this study, we have demonstrated that plasma NfL concentration is increased in patients with ATTRm related polyneuropathy and that it correlates with disease severity. This suggests pNfL may be a useful biomarker for monitoring disease progression and treatment efficacy.

The role of pNfL as a diagnostic biomarker in ATTRm related polyneuropathy is uncertain as it is raised in many other acquired, genetic, peripheral and central nervous system disorders, and increases with age ${ }^{5}$. However, the absence of a difference in the mean $\mathrm{pNfL}$ concentration between neurologically asymptomatic patients with ATTRm and controls suggests that pNfL may have utility in detecting the onset of neuropathy before clinical signs develop. This is of clinical relevance as several current treatments are only licenced for ATTRm patients with evidence of peripheral neuropathy.

Several invasive and non-invasive biomarkers have been assessed in ATTRm amyloidosis to aid in early diagnosis. Intraepidermal nerve fibre density (IENFD) and in vivo laser scanning confocal microscopy (IVCM) of the cornea sensitively detect small fibre dysfunction in patients with ATTRm and correlate with clinical severity measures ${ }^{11,12}$. IENFD is a quantitative, albeit invasive marker that would limit its use for repeated measurements and while IVCM is a non-invasive, rapid procedure, depending on the quantification method used, the results can be examiner dependent. High-resolution magnetic resonance neurography (MRN) has been shown to quantitively identify peripheral nerve impairment in sural nerves of asymptomatic and symptomatic ATTRm patients. This is an encouraging finding but has not been correlated with clinical parameters ${ }^{13}$.

There are a number of limitations to our study. Firstly, the number of control subjects is small, however, the pNFL concentrations in the cohort are in keeping with historical control samples from our group ${ }^{6}$. Secondly, a small proportion of our patients were prescribed 
diflusinal which may have a disease modifying effect and might artificially reduce pNFL concentration in the ATTRm cohort, however, the statistically significant results of this study are promising as NIS and CMTES-R were only available in a subset of patients, with a smaller subgroup having a neuropathy.

In this study, we have shown that pNfL concentration is sensitive to detecting peripheral axonal damage in ATTRm related polyneuropathy and that it correlates with two commonly used measures of disease severity. Longitudinal studies to assess responsiveness of pNfL to treatments and disease onset are needed before it can be adopted into routine clinical practice.

Acknowledgements:

MK gratefully received funding from a patient's bequest. AMR is funded by a Wellcome Trust Postdoctoral Fellowship for Clinicians (110043/Z/15/Z).

MMR is grateful to the Medical Research Council (MRC), MRC Centre grant (G0601943), and the National Institutes of Neurological Diseases and Stroke and office of Rare Diseases (U54NS065712) for their support. This research was also supported by the National Institute for Health Research University College London Hospitals Biomedical Research Centre. HZ has received funding from the European Research Council, the Swedish Research Council, the Wellcome Trust, the UK Dementia Research Institute at UCL, the Knut and Alice Wallenberg Foundation and the Leonard Wolfson Experimental Neurology Centre. MF has received funding from the Leonard Wolfson Experimental Neurology Centre. $\mathrm{AH}$ is funded by the Wolfson Foundation and the UK Dementia Research Institute. The Simoa instrument was bought using a Wellcome Trust multi-user equipment grant (PI: H.Z.). Study Funded by the NIHR UCLH Biomedical Research Centre Grant \#BRC279566.

Competing interests: 
MMR consults/serves on advisory boards for Alnylam Pharmaceuticals, Akcea Therapeutics, and Inflectis. AMR and MK have received support from Alnylam UK Limited to attend scientific meetings and an honorarium for speaking at a sponsored symposium. MMR has or has had consultancies with Alnylam Pharmaceuticals and Ionis Pharmaceuticals and MMR and ML were involved in the NEURO-TTR Trial (ClinicalTrials.gov Identifier: NCT01737398). HZ has served at advisory boards for CogRx, Samumed, Roche Diagnostics, Eli Lilly and Wave, has received travel support from Teva and is a co-founder of Brain Biomarker Solutions in Gothenburg AB, a GU Ventures-based platform company at the University of Gothenburg. JG serves on advisory boards for Alnylam Pharmaceuticals, Akcea Therapeutics, Pfizer Inc, and GlaxoSmithKline. The remaining authors declare that the research was conducted in the absence of any commercial or financial relationships that could be construed as a potential conflict of interest.

Conception and design: AMR, MMR

Provision of study materials or patients: AMR, MMR, JG, AM, MPL

Sample processing: $\mathrm{MF}, \mathrm{AH}, \mathrm{HZ}$

Data analysis and interpretation: AMR, MK

Manuscript writing: MK, AMR

Final approval of manuscript: All authors 


\begin{tabular}{|l|l|l|l|l|}
\hline Characteristics & Controls & All & ATTRm & ATTRm \\
& & & without & with \\
\hline No. & 16 & 85 & 6 & neurology \\
Age in years (median & $67(29.6-$ & $56.7(21.3-$ & $46.0(33.0-$ & $65.8(30.4-$ \\
(range)) & $80.2)$ & $80.6)$ & $60.6)$ & $80.3)$ \\
\hline Gender (F/M) & $1 / 14$ & $27 / 61$ & $3 / 3$ & $8 / 12$ \\
\hline Plasma NfL (pg.ml) & $15.5(11.6-$ & $58.1(45.1-$ & $2.5(0-5.2)$ & $68.4(35.1-$ \\
(mean (SD)) & $19.3)$ & $71.0)$ & & $101.8)$ \\
\hline
\end{tabular}

Table 1: Descriptive Statistics of Demographic and Clinical Variables in ATTRm Cohort and Controls

Figure 1: Plasma NfL concentration for all patients with ATTRm (TTR), healthy controls, ATTRm patients with neuropathy (Neuropathy) and ATTRm patients without neuropathy (No Neuropathy). $* \mathrm{p}<0.05 . \mathrm{ns}=$ not significant

Figure 2: Plasma NfL concentration plotted against disease severity as measured by the neuropathy impairment score (A), and the Rasch modified CMT examination score (B).

Figure 3: The neuropathy impairment score (NIS) plotted against the Rasch modified CharcotMarie-Tooth (CMT) examination score shows a significant correlation. 
1. Ando Y, Coelho T, Berk JL, Cruz MW, Ericzon BG, Ikeda SI, et al. Guideline of transthyretin-related hereditary amyloidosis for clinicians. Orphanet J Rare Dis [Accessed] 2013;8(1):31. 10.1186/1750-1172-8-31

2. Adams D, Gonzalez-Duarte A, O'Riordan WD, Yang C-C, Ueda M, Kristen A V., et al. Patisiran, an RNAi Therapeutic, for Hereditary Transthyretin Amyloidosis. N Engl $J$ Med 2018;379(1):11-21.

3. Benson MD, Waddington-Cruz M, Berk JL, Polydefkis M, Dyck PJ, Wang AK, et al. Inotersen Treatment for Patients with Hereditary Transthyretin Amyloidosis. $N$ Engl J Med [Accessed] 2018;379(1):22-31. 10.1056/NEJMoa1716793

4. Gertz MA. Immunoglobulin light chain amyloidosis: 2018 Update on diagnosis, prognosis, and treatment. Am J Hematol 2018;93(9):1169-80.

5. Khalil M, Teunissen CE, Otto M, Piehl F, Sormani MP, Gattringer T, et al. Neurofilaments as biomarkers in neurological disorders. Nat Rev Neurol [Accessed] 2018;14(10):577-89. 10.1038/s41582-018-0058-z

6. Sandelius Å, Zetterberg H, Blennow K, Adiutori R, Malaspina A, Laura M, et al. Plasma neurofilament light chain concentration in the inherited peripheral neuropathies. Neurology 2018;90(6):e518-24.

7. Sadjadi R, Reilly MM, Shy ME, Pareyson D, Laura M, Murphy S, et al. Psychometrics evaluation of Charcot-Marie-Tooth Neuropathy Score (CMTNSv2) second version, using Rasch analysis. J Peripher Nerv Syst 2014;19(3):192-6.

8. Kleyweg RP, Van Der Meché FGA, Schmitz PIM. Interobserver agreement in the assessment of muscle strength and functional abilities in Guillain-Barré syndrome. Muscle Nerve [Accessed] 1991 Nov;14(11):1103-9. 10.1002/mus.880141111

9. Dyck PJ, Litchy WJ, Lehman KA, Hokanson JL, Low PA, O’Brien PC. Variables influencing neuropathic endpoints: The Rochester Diabetic Neuropathy Study of 
Healthy Subjects. Neurology 2012;45(6):1115-21.

10. Norgren N, Toombs J, Fellows A, Woollacott IOC, Dick KM, Mead S, et al. Serum neurofilament light chain protein is a measure of disease intensity in frontotemporal dementia. Neurology 2016;87(13):1329-36.

11. Rousseau A, Cauquil C, Dupas B, Labbé A, Baudouin C, Barreau E, et al. Potential role of in vivo confocal microscopy for imaging corneal nerves in transthyretin familial amyloid polyneuropathy. JAMA Ophthalmol 2016;134(9):983-9.

12. Ebenezer GJ, Liu Y, Judge DP, Cunningham K, Truelove S, Carter ND, et al. Cutaneous nerve biomarkers in transthyretin familial amyloid polyneuropathy. Ann Neurol 2017;82(1):44-56.

13. Kollmer J, Sahm F, Hegenbart U, Purrucker JC, Kimmich C, Schönland SO, et al. Sural nerve injury in familial amyloid polyneuropathy. Neurology [Accessed] 2017 Aug 1;89(5):475-84. 10.1212/WNL.0000000000004178 